\title{
Numerical Investigation on Effect of Wrinkle on Stiffness in Isotropic Metal Sheets
}

\author{
S. Patil ${ }^{1 *}$, C. Ramadas ${ }^{2}$, R. Harshe ${ }^{2}$, S. Gurav ${ }^{1}$ \\ ${ }^{1 *}$ MTech Student, ${ }^{1}$ Associate Professor, Walchand College of Engineering, Sangli, India \\ ${ }^{2}$ Scientist F, ${ }^{2}$ Scientist E Composites Research Center, R\&DE(E), DRDO, Pune, India \\ \{supspatil2021@gmail.com\}
}

\begin{abstract}
A numerical study was conducted to find the effect of wrinkle in a metal sheet on its stiffness under tensile, compressive and flexural loading conditions. To define wrinkle of different sizes in model 'wrinkle factor' - ratio of thickness of specimen at wrinkle location to thickness of the specimen at no wrinkle location, is defined. Stiffness of a sheet with wrinkle has been compared with that of a pristine sheet under various loading conditions. From numerical results it is seen that, the stiffness in both tension and compression loading reduces as the wrinkle factor increases. But the stiffness in flexural loading is not affected considerably; contrary it increases for some wrinkle factor. Considering various loading conditions, it is observed that, stiffness in compression is affected more than in any other case. Furthermore, an attempt has been made to investigate force picked up by wrinkled portion and compared with the total force applied on the sample.
\end{abstract}

Keywords: Sheet metal, Wrinkle defect, Wrinkle factor, Modelling, Stiffness

\section{Introduction}

Even though the manufacturing technology is advanced, every manufacturing process has its own limitations due to defects, which occur during the process of manufacturing. Basically, wrinkle is a manufacturing defect, especially occurs in sheet forming process [1]. In non-metals also, for instance, manufacturing of fiber reinforced plastics [2], laminated structures [3], and thin curvature structures [4] formation of wrinkle takes place. Mostly wrinkle formation takes place in areas nearby corner region or in curvature region where member undergoes compression. This wrinkle formation in a structure depends on mode of pressurecoming over it and stiffness of the structure. If manufacturing pressure is not uniform or stiffness of member failed to sustain that pressure, then folds or wrinkle forms in the member.

Deep drawing is one of the important sheet forming processes. Besides its advantages, the limitations of this process are onset of wrinkling and fracture failure. One of the primary defects that occur in deep drawing operations is the wrinkling of sheet metal material, generally in the wall or flange of the part. The conditions for onset of wrinkling are controlled by the local geometry (sheet curvature) and current state of stress. Correia and Ferron [1] studied the onset of wrinkling in anisotropic metal sheets under deep drawing using an analytical approach and finite element solution. Bi-furcation analysis oncurved sheet elements subjectedto a biaxial stress state was carried out to investigate the effect of planar anisotropy on the onset of wrinkling in sheet metalforming process.

Investigation of Rajabi and Kadkhodayan [3] on deep drawing of fiber-metal laminates based on glassfiber reinforced polypropylene demonstrated that a high interaction between temperature and blank-holder force was required to remove wrinkle. Morovatiet al. [5] studied wrinkling of two layer (aluminium-stainless steel) sheets in deep drawing process through an analytical method, numerical simulation and experiments. Mohammad et al. [6] comparedthe effect of blank holder forces on wrinkling and fracture in deep drawing process of single and two-layer metal sheets and also, the effect of material on wrinkle

A lot of analysis on wrinkling based on functional and bifurcation criterion proposed by Hutchinson [7] for the study of plastic buckling was done. This criterion was first applied by Hutchinson and Neal [4] in a local wrinkling analysis for a doubly curved, elastic-plastic shell element submitted to a biaxial plane stress state. Rui Huang [8] presented a kinetic approach to wrinkling.

Most of the literature has explained the mechanism of formation of wrinkles in metal sheet, factors causing

B. Iyer, S. Nalbalwar and R. Pawade (Eds.)

ICCASP/ICMMD-2016. Advances in Intelligent Systems Research.

Vol. 137, Pp. 21-26.

(C) 2017. The authors - Published by Atlantis Press

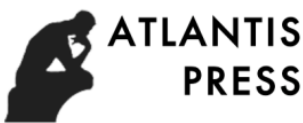

This is an open access article under the CC BY-NC license (http://creativecommons.org/licens)es/by-nc/4.0/). 
formation of wrinkle and gave criteria to control it. However, the effect of wrinkle formation on structural properties is not investigated enough. Hence, it is necessary to do quantitative study on the configuration of wrinkle formed, and the effect of wrinkle on strength and stiffness of the structure. Location and size of wrinkle formation is random generally at curved regions during the manufacturing of structure. Therefore, in this paper, analysis of wrinkle formed in a structure is done through numerical simulations, considering wrinkles of various sizes $1 / 4,1 / 2,3 / 4$ and $1 / 1$ compared to total sheet thickness and characterize their stiffness under tensile, compressive, and flexure loads.

\section{Numerical Modeling}

All numerical simulations in this paper were carried out using Finite Element Analysis (FEA) code, ANSYS.

\subsection{Modeling of Components}

Each model under loading conditions - tension, compression and flexure is explained hereunder. The magnitudes of tensile, compressive and flexural forces considered in the study were $90 \mathrm{kN}, 45 \mathrm{kN}$ and $30 \mathrm{kN}$, respectively. Table 1, listssizes of samples used in modellingunder three different loading conditions. Wrinkle wasintroduced based on thickness of plain specimen. As the name suggests, $1 / 4$ wrinkle specimen means, size of wrinkle is $1 / 4$ of the total thickness of the specimen. Inner and outer radii of wrinkle are selected according to wrinkling thickness of sample. Ratio atthickness of the specimen at wrinkle location to thickness of the specimen at no wrinkle location is termed as wrinkle factor. This factor increases with size of wrinkle. Sizes of the wrinkles considered in the modelling are given in Table 2.

Table 1 Dimensions of the samples under tensile, Compressive and Flexural loading

\begin{tabular}{|c|c|c|c|}
\hline Dimension & $\begin{array}{c}\text { Tensile } \\
\text { Loading }\end{array}$ & $\begin{array}{c}\text { Compressive } \\
\text { Loading }\end{array}$ & $\begin{array}{c}\text { Flexural } \\
\text { Loading }\end{array}$ \\
\hline Width $(\mathrm{mm})$ & 20 & 20 & 20 \\
\hline Length(mm) & 350 & 160 & 300 \\
\hline Thickness $(\mathrm{mm})$ & 20 & 20 & 20 \\
\hline
\end{tabular}

FE model was generated using 2D SOLID element SOLID182, which is a four node element. Material assumed was aluminium, whose properties are listed in Table 3. Figure 1 shows a pristine specimen. Specimens with wrinkle of different sizes are shown in Figure 2. Size of element in FE mesh was $2 \mathrm{~mm}$. However, fine mesh was generated at wrinkle area.

Table 2 Dimensions of various wrinkles considered in modeling

\begin{tabular}{|l|l|l|l|l|l|}
\hline \multicolumn{1}{|c|}{ Samples } & $\begin{array}{c}\text { Inner radius } \\
(\mathbf{m m})\end{array}$ & $\begin{array}{c}\text { Outer radius } \\
(\mathbf{m m})\end{array}$ & $\begin{array}{c}\text { Plain } \\
\text { Thickness } \\
(\mathbf{t p}-\mathbf{m m})\end{array}$ & $\begin{array}{c}\text { Thickness } \\
\text { at Wrinkle } \\
(\mathbf{t w}-\mathbf{m m})\end{array}$ & $\begin{array}{c}\text { Wrinkle factor } \\
\text { tw/tp }\end{array}$ \\
\hline Plain & - & - & 20 & 20 & 1 \\
\hline $1 / 4$ wrinkle & 5 & 10 & 20 & 25 & 1.25 \\
\hline $1 / 2$ wrinkle & 10 & 20 & 20 & 30 & 1.5 \\
\hline 3/4 wrinkle & 15 & 30 & 20 & 35 & 1.75 \\
\hline Full wrinkle & 20 & 40 & 20 & 40 & 2 \\
\hline
\end{tabular}

Table 3 Mechanical properties of Aluminium metal

\begin{tabular}{|c|c|c|}
\hline Young's Modulus (GPa) & Poisson's Ratio & Density $\left(\mathbf{K g} / \mathbf{m}^{\mathbf{3}}\right)$ \\
\hline 69 & 0.33 & 2700 \\
\hline
\end{tabular}




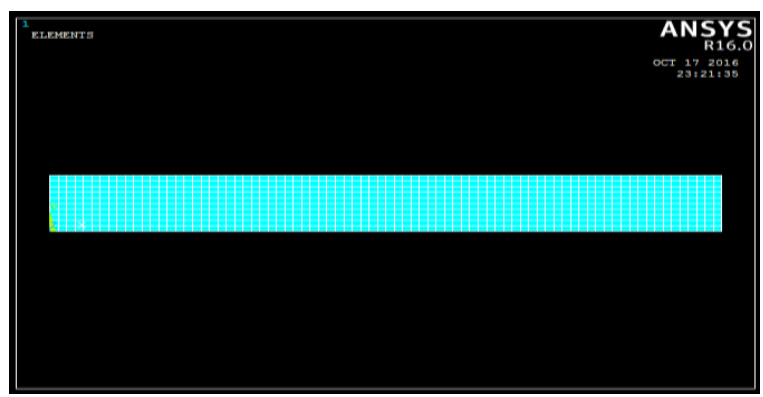

Fig. 1. Model of plain specimen without wrinkle

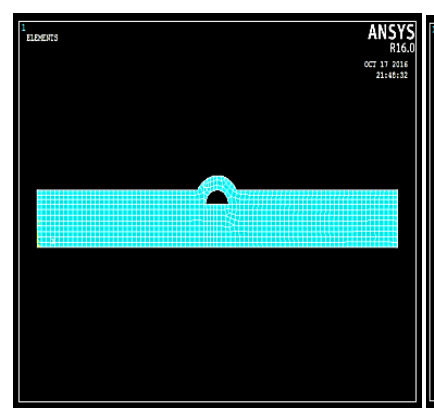

a) $1 / 4$ wrinkle

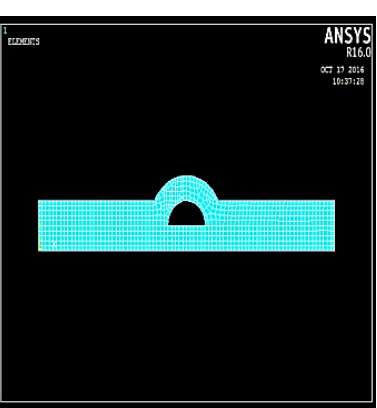

b) $1 / 2$ wrinkle

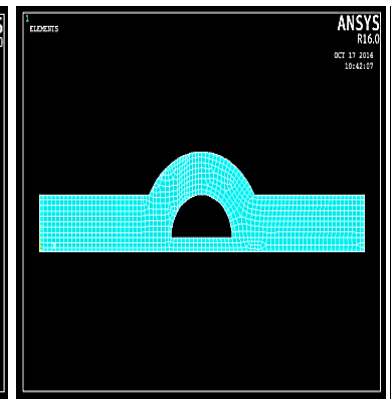

c) $3 / 4$ wrinkle

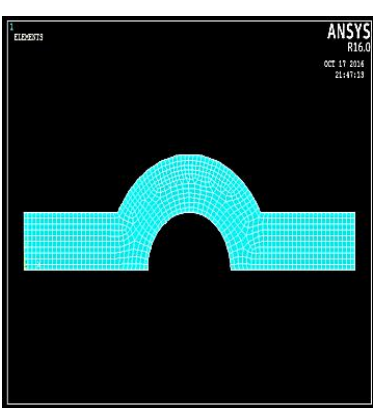

d) Full wrinkle

Fig. 2. Models of various wrinkle considered

\subsection{Modelling of Compressive Loading}

Compressive loading was applied to estimate stiffness of specimen under compressive loading. Boundary Conditions (BCs) and loading applied at both ends of specimen are as shown in Fig. 3. Degree of Freedom (DoF) in $x$ direction is coupled to have uniform compression/ deformation. Static Analysis wasperformed under the above loading and BCs. Estimated stiffness under compressive load is presented graphically in Fig. 6(a).

\subsection{Modelling of Tensile Loading}

BCs applied under tensile load were same as those discussed in compression.

Fig. 4 shows the BCs and loading on the specimen. Graphical representation of stiffness under tensile load is shown in Fig. 6(b).

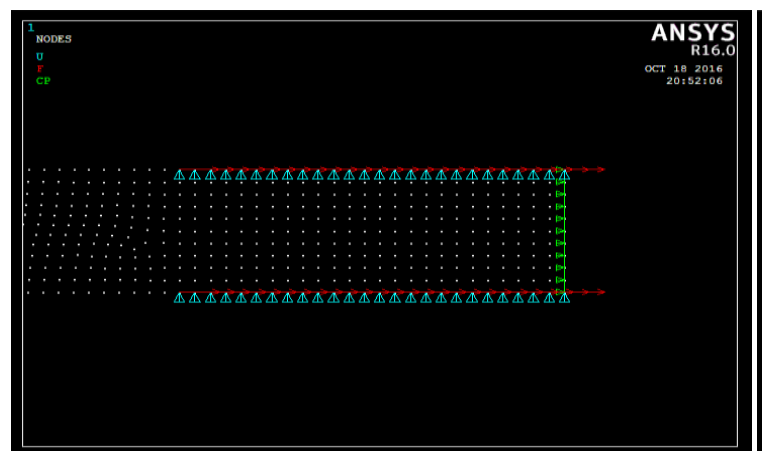

Fig. 3. Loading and boundary condition in compression

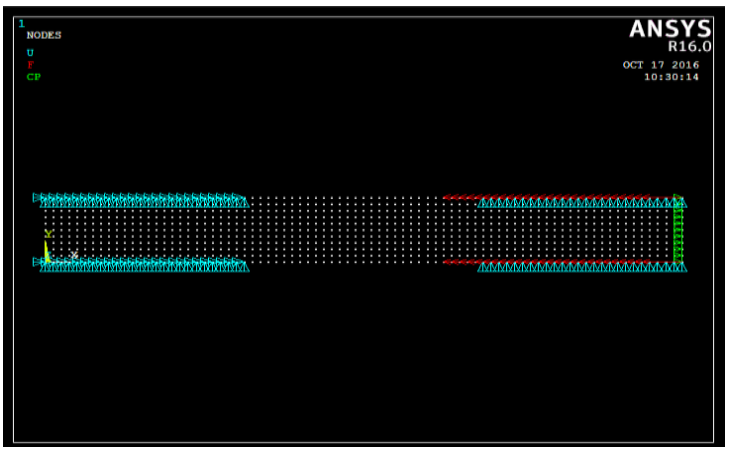

Fig. 4. Loading in tensile testing 


\subsection{Modelling of FlexuralLoading}

Flexure stiffness was predicted under four point bend test [9]. Flexural stiffness was calculated in two modes (a) wrinkle on load side and (b) wrinkle on opposite side to load side, as shown in Fig. 5, Fig. 6(c) shows flexural stiffness obtained in both the cases.

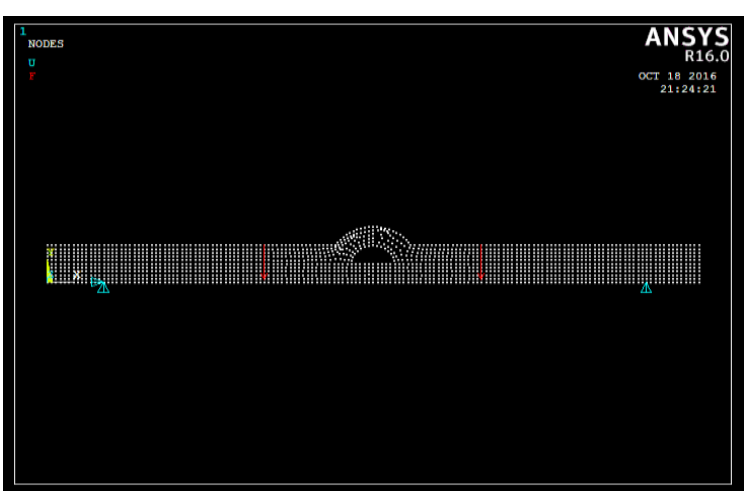

a) Wrinkle on load side

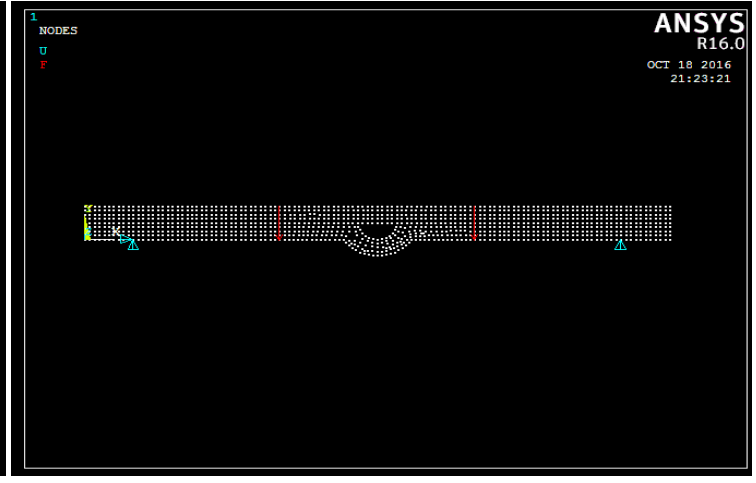

b) Wrinkle on opposite to load side

Fig. 5. Loading and boundary condition applied in Flexural test

\section{Results and Discussion}

\subsection{Effect on stiffness due to wrinkles}

Comparison of stiffness of wrinkled specimen is done with pristine specimen. As compared to stiffness of pristine specimen, stiffness of specimens with wrinkles goes on decreasing with increase in wrinkle factor under compressive and tensile loading. There is no significant difference in stiffness of pristine specimen and specimen with wrinkle factor 1.25. Figure 6 shows variation in stiffness with wrinkle factor under various loading conditions. Compressive and tensile stiffness decreases predominantly from wrinkle factor 1.5. From flexural stiffness results, it is inferred that, stiffness of specimens in both wrinkle on load side and wrinkle on opposite side are nearly same. Reduction in stiffness at 1.25 wrinkle factor is negligible when compared stiffness of pristine specimen. However, there is an increase in stiffness of the specimen at 1.5 and 1.75 wrinkle factors. Here it is conjectured that $1 / 2$ and $3 / 4$ wrinkles are acting as stiffeners in flexural loading. When a full wrinkle was introduced, stiffness has reduced by $20 \%$. This could be because, except wrinkle, there is no material, which bridges the gap between two parts of the specimen. Therefore, under this configuration, specimen loses its stiffness.

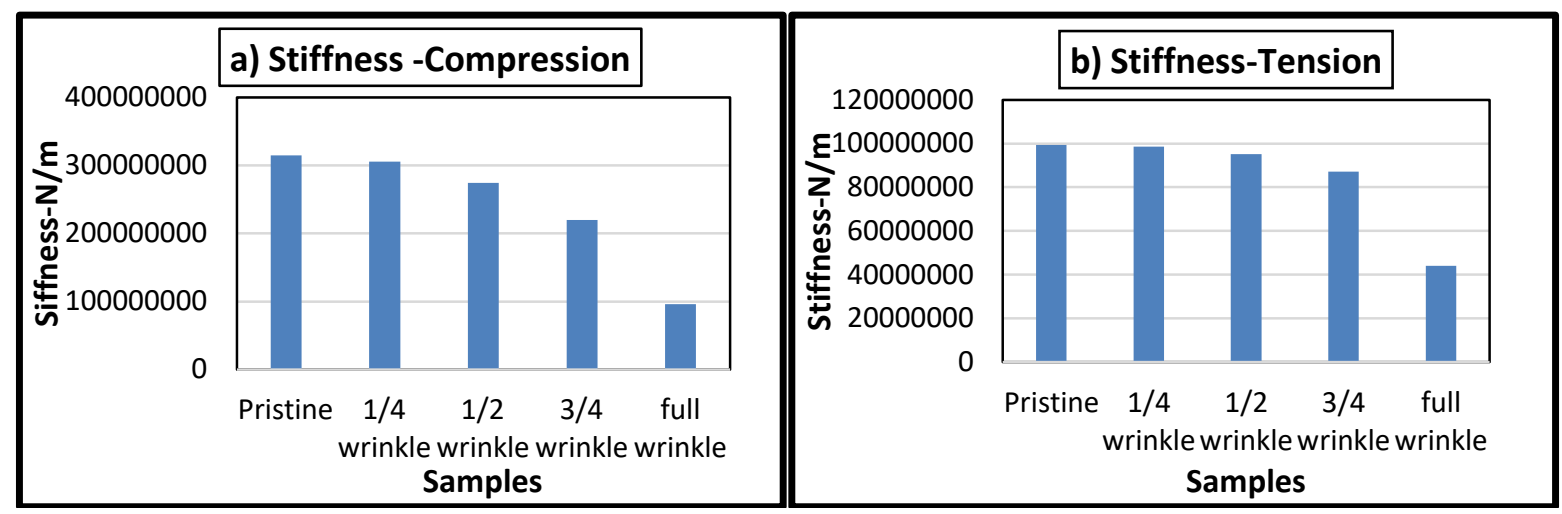




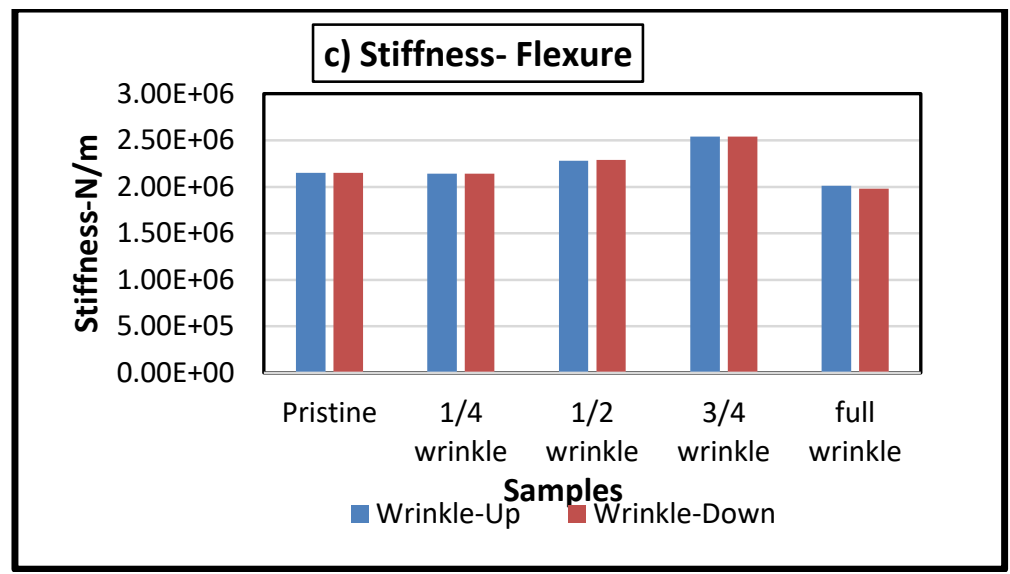

Fig. 6. Graphical presentation of stiffness for all specimens

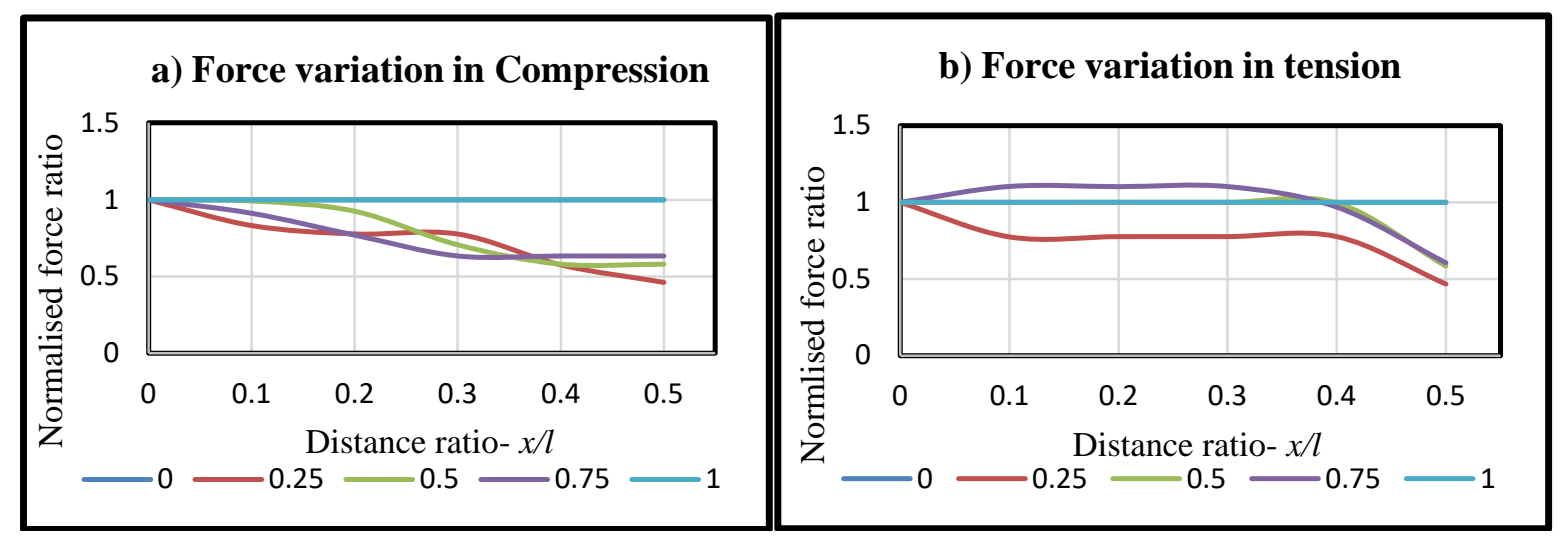

Fig. 7. Graphical presentation of force variation in wrinkled thickness of specimens

\subsection{Force variation across all specimens under compressive, tensile and flexural loading conditions}

Force transmitted to wrinkle portion at sections across the half-length was normalised with force carried by wrinkle thickness at section $(x / l=0)$ where wrinkle effect is negligible, is listed in Fig. 7. This was performed for all wrinkle factors under tensile and compressive loadings. Normalised force ratio decreases as the distance ratio increases; this is true for all specimens under tensile and compressive loading conditions. In Fig. 7, percentage reduction of load carried by wrinkle $(x / l=0.5)$ with respect to load carried by specimen section at $x / l=0$ is also shown.

\section{Conclusions}

As wrinkle factor increases, stiffness of specimen decreases in compressive and tensile loading conditions. This effect is more prominent when wrinkle factor is more than 1.5. Effect of wrinkle having 1.25 factor on stiffness is not significant, when compared to the stiffness of specimens with higher wrinkle factors. In flexural loading, there is no detrimental effect on stiffness due to wrinkle, when the factor is in between 1.25 to 1.75 , on the contrary the wrinkle enhances flexural stiffness. At the position of wrinkle, the load carried by wrinkle with wrinkle factors $1.25,1.5,1.75$ and 1 is $14 \%, 28 \%, 47 \%$ and $100 \%$ of total load respectively. There is no reduction in load carried by wrinkle with wrinkle factor 1 because, as the whole thickness is wrinkled, there isonly one way i.e. wrinkle, for load transfer. Even though the full wrinkle takes the total load, its effect on stiffness is hostile. Effect of wrinkle with wrinkle factor one on stiffness is more than other wrinkle factors.As the wrinkle size and position of wrinkle formation is arbitrary, it is difficult to pre-consider the dimension of wrinkle. In this paper, inner radius of wrinkle considered was based on thickness of the wrinkle. The results can change with wrinkle's inner and outer radius too. 


\section{Acknowledgement}

First author sincerely thanksall officers and staff working at Composite Research Centre in R\&DE(E)for their guidance and timely support as well as the administrative staff for providing me an opportunity to work with Scientists of prestigious research institute.

\section{References}

[1] J. P. De Magalhaes Correia, G. Ferron., "wrinkling of anisotropic metal sheets under deep-drawing: analytical and numerical study", Journal of Materials Processing Technology, 155-156 (2004) 16041610.

[2] P. Hallander, M. Akermo, C. Mattei, M. Petersson, T. Nyman, "An experimental study of mechanisms behind wrinkle development during forming of composite laminates”, Composites: Part A, 50(2013) 5464.

[3] J. W. Hutchinson, K. W. Neale, " Wrinkling of curved thin sheet metal", Proceedings of International Symposium on Plastic Instability, Considere Memorial: Presses des Ponts et Chaussees; (1985) 71-80.

[4] A. Rajabi, M. Kadkhodayan, "An Investigation into the Deep Drawing of Fiber-metal Laminates based on Glass Fiber Reinforced Polypropylene", International Journal of Engineering, 2(3) (2014) 349-358.

[5] M. R Morovvati, B. M. Dariani, M. H. Asadian, " A theoretical, numerical, and experimental investigation of plastic wrinkling of circular two-layer sheet metal in the deep drawing", Journal of materials processing Technology, 210(2010) 1738-1747.

[6] M. R. Morovvati, A. Fatemi, M. Sadighi, "Experimental and finite element investigation on wrinkling of circular single layer and two-layer sheet metals in deep drawing process", International Journal of Advanced Manufacturing Technology, 54(1) (2011) 113-121.

[7] J. W. Hutchinson, Advances in applied mechanics, Chia-Shun Yih, New York: Academic Press; 14(1974) 67-144.

[8] Ruihuang, "A Kinetics Approach to Surface Wrinkling of Elastic thin films".

[9] R.C. Hibblelar, Mechanics of materials, second ed., Prentice Hall, 2005. 